\title{
Hecke Basis Theorems for Groups of Genus 0*
}

\author{
Marvin I. Knopp** and John Roderick Smart**
}

(May 1, 1970)

\begin{abstract}
A Hecke-type basis theorem is established for the cusp forms of negative even integral degree (multiplier system 1) on the class of Hecke groups. Hecke established the result for the classical modular group, which is the first of the Hecke groups. A second result is a parametrization theorem for entire automorphic forms of negative real degree (with arbitrary multiplier systems) on certain discrete groups of real linear fractional transformations of genus zero.
\end{abstract} forms.

Key words: Eisenstein series; Hecke group; H-group; modular form; parametrization of cusp

\section{Introduction}

In [11, Theorem 2] ${ }^{1}$ Rademacher and Zuckerman establish a "parametrization theorem" for modular forms of all real degrees and as a consequence give an explicit parametric representation for all multiplier systems connected with the modular group $\Gamma(1)$. In a different (but related) direction, Hecke [1, p. 3] earlier established the existence of a particularly useful special type of basis for the cusp forms of negative even integral degree with multiplier system identically 1 on $\Gamma(1)$; this basis is given in terms of the well-known modular form

and the "Eisenstein series"

$$
\Delta(z)=e^{2 \pi i z} \prod_{m=1}^{\infty}\left(1-e^{2 \pi i m z}\right)^{24}
$$

$$
E_{k}(z)=\sum(c z+d)^{-k},
$$

where $k$ is an even integer $\geqslant 4$ and the summation is on all pairs of relatively prime rational integers $c, d . \Delta(z)$ is a cusp form of degree -12 and multiplier system identically 1 on $\Gamma(1)$, whereas $E_{k}(z)$ is an entire modular form of degree $-k$ and multiplier system 1 , but not a cusp form since $E_{k}(z) \rightarrow 2$ as $z \rightarrow i \infty$. It is clear from the representation (1.1) that $\Delta(z)$ has no zeros in $\mathscr{H}$, the upper half-plane.

Theorem 1 of this article is a Hecke-type basis theorem for the cusp forms of negative even integral degree with multiplier system 1 on an infinite class of discrete groups $\Gamma\left(\lambda_{n}\right), n$ an integer $\geqslant 3$, of linear fractional transformations preserving $\mathscr{H} . \Gamma\left(\lambda_{n}\right)$, called the $n$th Hecke group (or the Hecke group of parameter $n$ ), is the group generated by the two matrices

$$
S_{n}=\left(\begin{array}{ll}
1 & \lambda_{n} \\
0 & 1
\end{array}\right), \quad T=\left(\begin{array}{rr}
0 & -1 \\
1 & 0
\end{array}\right),
$$

with $\lambda_{n}=2 \cos (\pi / n)$. Here we have adopted the convention, to which we adhere throughout, that the matrix $M=\left(\begin{array}{ll}a & b \\ c & d\end{array}\right)$ is to be identified with the linear fractional transformation

$$
z \rightarrow(a z+b) /(c z+d)=M z .
$$

Note that $-M$ is then identified with the same linear fractional transformation. In particular the matrix $-I$ is the identity when thought of as a linear fractional transformation. $\Gamma(1)$, the modular group, is the Hecke group of parameter 3 ; that is $\Gamma(1)=\Gamma\left(\lambda_{3}\right)$, since $\Gamma(1)$ is generated by

*An invited paper

**Department of Mathematics, University of Wisconsin, Madison, Wis. 53706.

' Figures in brackets indicate the literature references at the end of this paper. 


$$
U=S_{3}=\left(\begin{array}{ll}
1 & 1 \\
0 & 1
\end{array}\right) \quad \text { and } \quad T=\left(\begin{array}{rr}
0 & -1 \\
1 & 0
\end{array}\right)
$$

Our second principal result, given in section 7 and summarized in table 2, is a parametrization theorem for entire automorphic forms of negative real degree on certain discrete groups of linear fractional transformations acting on $\mathscr{H}$, of genus zero. Among the groups included are $\Gamma(1) ; \Gamma_{\vartheta}$, the group connected with the well-known modular form $\vartheta(z)=\vartheta_{3}(0 \mid z)$ and generated by

$$
U^{2}=\left(\begin{array}{ll}
1 & 2 \\
0 & 1
\end{array}\right) \quad \text { and } \quad T=\left(\begin{array}{rr}
0 & -1 \\
1 & 0
\end{array}\right)
$$

$\Gamma(2)$ and $\Gamma(3)$, the principal congruence subgroups of levels 2 and 3 (For $n$ a positive integer $\Gamma(n)$ is the normal subgroup of $\Gamma(1)$ defined by the congruence condition

$$
\left.\left(\begin{array}{ll}
a & b \\
c & d
\end{array}\right) \equiv \pm \mathrm{I}(\bmod n) .\right)
$$

$\Gamma\left(\lambda_{4}\right)=\Gamma(\sqrt{2})$ and $\Gamma\left(\lambda_{6}\right)=\Gamma(\sqrt{3})$. For these groups the entire forms are parametrized and the multiplier systems are explicitly determined. Other groups of genus zero are also included in table 2, but the results are neither as complete nor as explicit as in the cases listed above. It is of interest to note that the multiplier systems connceted with entire forms of negative degree on a discrete group $\Gamma$ include all the multiplier systems connected with $\Gamma$. Thus in those cases where we explicitly calculate the multiplier systems for entire forms, we have in fact determined all multiplier systems.

The parametrization theorem of Rademacher and Zuckerman expresses all modular forms (of all degrees) in terms of a small number (four) of familiar modular functions and forms. In contrast, our results of section 7 express all entire forms of a fixed degree $-r$ in terms of a finite set of entire forms of degree $-r$; the number of functions in the set equals the dimension of the space of entire forms of degree $-r$ and therefore increases with $r$. Essentially the same types of functions occur here as in theorem 1 and we would therefore be justified in referring to the results of section 7 as "Hecke basis theorems", generalized to include the treatment of entire forms of arbitrary negative real degree, with arbitrary multiplier systems.

The function $\Delta(z)$ defined by (1.1) plays an important role in Hecke's original basis theorem as it has no zeros in $\mathscr{H}$ and a zero of order 1 (in the uniformizing variable $e^{2 \pi i z}$ ) at $i \infty$. To obtain our basis theorem for the Hecke groups $\Gamma\left(\lambda_{n}\right)$, we need a cusp form on $\Gamma\left(\lambda_{n}\right)$ with similar properties. In section 3 we show that, for an arbitrary $H$-group $\Gamma$ of genus 0 , there exists a cusp form $\Delta(\Gamma, r, 1 ; z)$ of negative even integral degree $-r$ and multiplier system 1 with no zeros in $\mathscr{H}$. An appropriate power of $\Delta(\Gamma, r, 1 ; z)$ will then be a cusp form with a zero of order exactly 1 at $i \infty$. In the case of the group $\Gamma\left(\lambda_{n}\right)$ we denote this cusp form $\Delta_{1}(z ; n)=\Delta_{1}(z)$. As might be expected $\Delta_{1}(z ; 3)=\Delta(z)$, the function defined by $(1.1)$.

An explicit statement of theorem 1 requires the introduction of Eisenstein series in a more general sense than that of (1.2). In fact Eisenstein series can be defined for all $H$-groups and in more general situations as well. In section 2 we give the definition of the Eisenstein series

$$
E_{r}(\Gamma, v ; z)
$$

an entire automorphic form of real degree $-r \leqslant-2$, with multiplier system $v$, on an arbitrary $H$-group $\Gamma$. In the case $r=2$ convergence difficulties present themselves and the Eisenstein series $E_{2}(\Gamma, v ; z)$ can be defined only if $v \equiv 1$. For notational convenience we also define $E_{0}(\Gamma, v ; z)=1$. With $k$ an even integer $\geqslant 4$ consider the functions

$$
B_{l}=E_{k-4 n l /(n-2)}\left(\Gamma\left(\lambda_{n}\right), v_{1}^{-1} ; z\right) \cdot \Delta_{1}^{l}(z), \quad 0<l<(k-2)(n-2) / 4 n,
$$

where $l$ is understood to be an integer and $v_{1}$ is the multiplier system of the cusp form $\Delta_{1}(z)$. 
The upper inequality is imposed upon $l$ to insure that $k-4 n l /(n-2)>2$. We also include the function

$$
B_{l}=E_{2}\left(\Gamma\left(\lambda_{n}\right), v_{1}^{-l} ; z\right) \cdot \Delta_{1}^{l}(z), \quad l=(k-2)(n-2) / 4 n,
$$

if $4 n \mid(k-2)(n-2)$ and $v_{1}^{l} \neq=1$. Finally we consider the function

$$
B_{l}=E_{0}\left(\Gamma\left(\lambda_{n}\right), v_{1}^{-l} ; z\right) \cdot \Delta_{1}^{l}(z), \quad l=k(n-2) / 4 n .
$$

if $4 n \mid k(n-2)$ and $v_{1}^{l} \equiv 1$. The functions $B_{l}$ of (1.3), (1.4), and (1.5) are all cusp forms of degree $-k$ with multiplier system 1 on $\Gamma\left(\lambda_{n}\right)$, and furthermore they are linearly independent since $B_{l}$ has a zero at $i \infty$ of order exactly $l$. The question of when they form a basis for the vector space of such cusp forms is dealt with in theorem 1 which follows.

THEOREM 1: Let $\mathrm{k}$ be an even integer $\geqslant 4$. With $\mathrm{n}$ an integer $\geqslant 3$, write $\mathrm{k}=4 \mathrm{~ns}+2 \mathrm{p}, \mathrm{s}$ an integer $\geqslant 0$ and $0 \leqslant \mathrm{p}<2 \mathrm{n}$. Then the functions $\mathrm{B}_{l}$ form a basis for the complex vector space of cusp forms of degree - $\mathrm{k}$ with multiplier system 1 on the group $\Gamma\left(\lambda_{\mathrm{n}}\right)$ except in the cases

$$
\frac{\mathrm{n}}{2}+\mathrm{l}<\mathrm{p}<\mathrm{n}, \quad \text { p even }
$$

and

$$
\frac{3 \mathrm{n}}{2}+1<\mathrm{p}<2 \mathrm{n}, \quad \text { p even. }
$$

In the exceptional cases (1.6) and (1.7) the number of functions $\mathrm{B}_{1}$ is 1 less than the dimension of the space of cusp forms.

REMARKs: 1. $B_{l}$ has a zero of order exactly $l$ (in the uniformizing variable $e^{2 \pi i z / \lambda_{n}}$ ) at $i \infty$. This property of the set of functions $B_{l}$ is the property that justifies our calling the set of $B_{l}$ 's a "Hecke basis" in those cases when it indeed is a basis.

2. In the exceptional cases (1.6) and (1.7) the "missing function" is the one with highest possible order zero at $i \infty$.

3. If $n=3,4$, or 6 there are no integers $p$ satisfying (1.6) or (1.7). Thus in these cases a Hecke basis is always achieved. The groups in question are $\Gamma(1), \Gamma(\sqrt{2})$, and $\Gamma(\sqrt{3})$, respectively. In particular, then, Hecke's original basis theorem is contained in theorem 1 .

The proof of theorem 1 is given in section 5, while section 6 is devoted to a short discussion of the missing function in the exceptional cases (1.6) and (1.7). In section 4 a Hecke basis theorem is given in the case of a restricted class of $H$-groups for which a very simple proof suffices. Among the groups included there are $\Gamma(1)$ and $\Gamma(\sqrt{2})$ again.

\section{Preliminary Definitions and Notation}

An $H$-group $\Gamma$ is a discrete subgroup of $S L(2, R)$ (thought of as linear fractional transformations), where $R$ is the real numbers, such that

(i) $\Gamma$ is finitely generated,

(ii) $\Gamma$ contains translations,

and

(iii) every point of the real axis is a limit point for $\Gamma$.

To put this another way, an $H$-group is a finitely generated Fuchsian group of the first kind, with translations, acting on $\mathscr{H}$. Such a group has a presentation of the form [7, p. 173]

$$
\begin{aligned}
& \left\{A_{1}, B_{1}, \ldots, A_{g}, B_{g}, E_{1}, \ldots, E_{e}, P_{1}, \ldots, P_{t},-I ;(-I)^{2}=I\right. \text {, }
\end{aligned}
$$

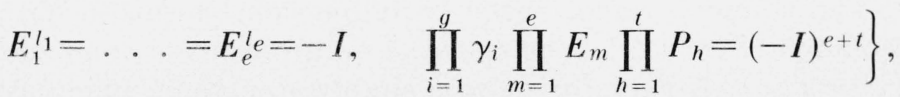

where $l_{m}, 1 \leqslant m \leqslant e$, is an integer $\geqslant 2, \gamma_{i}=A_{i} B_{i} A_{i}^{-1} B_{i}^{-1}, 1 \leqslant i \leqslant g$, the $A_{i}$ and $B_{i}$ are hyperbolic 
matrices, the $E_{m}$ are elliptic matrices, the $P_{h}$ are parabolic matrices, and $I$ is the $2 \times 2$ identity matrix. The numbering is so arranged that $P_{t}=\left(\begin{array}{ll}1 & \lambda \\ 0 & 1\end{array}\right), \lambda>0$, a translation by the real number $\lambda$. When the group $\Gamma$ has the presentation $(2.1)$ we say that $\Gamma$ is of signature $\left(g, n ; l_{1}, \ldots ., l_{e}\right)$, where $n=e+t$. The integer $g$ is called the genus of $\Gamma$. In particular, the modular group $\Gamma(1)$ is of signature $(0,3 ; 2,3)$, with generating matrices

$$
E_{1}=-T=\left(\begin{array}{rr}
0 & 1 \\
-1 & 0
\end{array}\right), \quad E_{2}=\left(\begin{array}{rr}
0 & 1 \\
-1 & 1
\end{array}\right), \quad P_{1}=U=\left(\begin{array}{ll}
1 & 1 \\
0 & 1
\end{array}\right) .
$$

In this article we shall be concerned only with $H$-groups $\Gamma$ of genus $g=0$, so we may assume that $\Gamma$ has the presentation

$$
\left\{E_{1}, \ldots, E_{e}, P_{1}, \ldots, P_{t} ; E_{1}^{l}=\ldots=E_{e}^{l}=-I, \prod_{m=1}^{e} E_{m} \prod_{h=1}^{t} P_{h}=(-I)^{e+t}\right\} .
$$

Let $r$ be a real number and let $\Gamma$ be an $H$-group. We want to consider functions $F(z)$, meromorphic in $\mathscr{H}$, such that

$$
F(V z)=v(V)(c z+d)^{r} F(z)
$$

for every $V=\left(\begin{array}{ll}a & b \\ c & d\end{array}\right) \epsilon \Gamma$ and $z$ in $\mathscr{H}$. Here $v(V)$ is a complex number of absolute value 1 independent of $z$. In order to fix the branch of $(c z+d)^{r}$ when $r$ is not an integer, we define, for any complex number $\tau$ and real number $s$,

$$
\tau^{s}=|\tau|^{s} \cdot \exp (i \arg \tau), \quad-\pi<\arg \tau \leqslant \pi
$$

When such a function $F(z) \neq \equiv$ exists, it follows that

$$
v\left(V_{1} V_{2}\right)\left(c_{3} z+d_{3}\right)^{r}=v\left(V_{1}\right) v\left(V_{2}\right)\left(c_{1} V_{2} z+d_{1}\right)^{r}\left(c_{2} z+d_{2}\right)^{r},
$$

where $V_{i}=\left(\begin{array}{ll}* & * \\ c_{i} & d_{i}\end{array}\right) \epsilon \Gamma$ for $i=1,2$ and $V_{1} V_{2}=\left(\begin{array}{ll}* & * \\ c_{3} & d_{3}\end{array}\right)$. A function $v$ from $\Gamma$ to the complex numbers of absolute value 1 and satisfying the consistency condition (2.5) is called a multiplier system for $\Gamma$ of degree $-r$.

Each parabolic generator $P_{h}$ of $\Gamma$ has a single fixed point and it is real. This point $x_{h}$ is called a parabolic cusp; the stabilizer of $x_{h}$ in $\Gamma$ is the cyclic subgroup of $\Gamma$ generated by $P_{h}, 1 \leqslant h \leqslant t$. From this latter fact, equation (2.3), and the fact that $F(z)$ is meromorphic in $\mathscr{H}$ it follows [4, pp. 272-273] that $F(z)$ has an expansion "at $x_{h}$ " of the form

$$
F(z)=\left(z-x_{h}\right)^{-r} \sum_{n=-\infty}^{\infty} b_{n}(h) e^{2 \pi i\left(n+\kappa_{h}\right)\left(A_{h} z\right) / \lambda_{h}}
$$

where $\lambda_{h}>0, A_{h}$ is a real linear fractional transformation such that $A_{h}\left(x_{h}\right)=\infty$, and $\kappa_{h}$ is defined by $v\left(P_{h}\right)=e^{2 \pi i \kappa_{h}}, 0 \leqslant \kappa_{h}<1$.

Suppose that $F(z)$ is meromorphic in $\mathscr{H}$, satisfies (2.3), and for each $h, 1 \leqslant h \leqslant t$, only finitely many terms such that $n<0$ appear in (2.6). Then we say that $F(z)$ is an automorphic form of degree $-r$ with respect to the group $\Gamma$ and the multiplier system $v$. If $F(z)$ is holomorphic in $\mathscr{H}$ and for each $h$ only terms such that $n+\kappa_{h} \geqslant 0$ appear in (2.6) we say that $F(z)$ is an entire automorphic form. If $F(z)$ is an entire form such that only terms with $n+\kappa_{h}>0$ appear in (2.6) we say that $F(z)$ is a cusp form. The complex vector space of automorphic forms of degree $-r$ with multiplier system $v$ on $\Gamma$ is denoted $\{\Gamma,-r, v\}$. The space of entire forms is denoted $C^{+}(\Gamma,-r, v)$ and that of cusp forms $C^{0}(\Gamma,-r, v)$. A modular form is simply an automorphic form with respect to the modular group $\Gamma(1)$. 
Besides the expansions (2.6) at the parabolic cusps (and of course the usual Taylor or Laurent expansions at points of $\mathscr{H}$ ), an automorphic form $F(z)$ in $\{\Gamma,-r, v\}$ has special expansions at the fixed points $\omega_{m}$ in $\mathscr{H}$ of the elliptic transformations $E_{m}, 1 \leqslant m \leqslant e[7$, pp. 180-181]:

$$
F(z)=\left(z-\bar{\omega}_{m}\right)^{-r} \sum_{n=\nu_{m}}^{\infty} b_{n}\left(\omega_{m}\right) u^{n+a_{m} / l_{m}}
$$

where $l_{m}$ is the order of $E_{m}$ in the sense of $(2.2), u=\left\{\left(z-\omega_{m}\right) /\left(z-\bar{\omega}_{m}\right)\right\}^{l_{m}}$, and

$$
v\left(E_{m}\right)=e^{\pi i r / l_{m}+2 \pi i a_{m} / l_{m}}, \quad 0 \leqslant a_{m} \leqslant l_{m}-1,
$$

with $a_{m}$ an integer. Now (2.6) and (2.7) enable us to define the order of $F(z)$ at all points (including the parabolic cusps) of a fundamental region $\mathscr{R}$ for $\Gamma$ in $\mathscr{H}$. The order of $F(z)$ at points of $\mathscr{H}$ not fixed by elliptic transformations in $\Gamma$ is defined in the usual way. $\mathscr{R}$ can be so constructed that the only points in $\mathscr{R}$ fixed by elliptic transformations in $\Gamma$ are the points $\omega_{m}$ fixed by the transformations $E_{m}, 1 \leqslant m \leqslant e$. At $\omega_{m}$ the order of $F(z)$ is defined to be $\nu_{m}+a_{m} / l_{m}$. Finally suppose that in (2.6) the nonzero $b_{n}(h)$ with smallest subscript occurs for $n=n_{h}$. Then we define the order of $F(z)$ at the parabolic cusp $x_{h}$ to be $n_{h}+\kappa_{h}$. With these definitions the sum of orders of $F(z) \epsilon\{\Gamma,-r, v\}$ at points in $\mathscr{R}$ is $r \cdot\left(g-1+\frac{q}{2}\right)$, where

$$
q=t+\sum_{m=1}^{e}\left(1-\frac{1}{l_{m}}\right)
$$

and $g$ is the genus of $\Gamma[6, \mathrm{p} .191]$. This result can be proved, for example, by integrating $F^{\prime}(z) / F(z)$ over the boundary of $\mathscr{R}$. In particular, if $F(z) \epsilon C^{+}(\Gamma,-r, v)$, then the total number $N$ of zeros of $F(z)$ in $\mathscr{R}$ is given by the formula

$$
N=r\left(g-1+\frac{q}{2}\right)
$$

Note that $N$ need not be an integer.

We next turn to the promised definition of the Eisenstein series $E_{r}(\Gamma, v ; z)$. Suppose, as before, that in the presentation (2.2) of $\Gamma$ the numbering is arranged so that $P_{t}=\left(\begin{array}{cc}1 & \lambda \\ 0 & 1\end{array}\right), \lambda>0$. Let $v$ be a multiplier system for $\Gamma$ of degree $-r$ such that, in addition, $v\left(P_{t}\right)=1$; this is equivalent to assuming that $\kappa_{t}=0$. If $r \geqslant 2$, then put

$$
E_{r}(\Gamma, v ; z)=\sum \bar{v}(V)(c z+d)^{-r},
$$

where the summation is over all elements $V=\left(\begin{array}{ll}* & * \\ c & d\end{array}\right)$ with distinct lower rows. It can be shown that since $v\left(P_{t}\right)=1, v(V)$ is independent of the upper row of $V$ and therefore (2.11) is unaffected by the choice of $V$ with lower row $c, d$. If $r>2$, then the series in (2.11) converges absolutely. From the absolute convergence and (2.5) it is easy to deduce that $E_{r}(\Gamma, v ; z) \epsilon C^{+}(\Gamma,-r, v)$. If $r=2$ then there are convergence difficulties which have been overcome by Petersson. In this case the series (2.11), suitably interpreted, is in $C^{+}(\Gamma,-2, v)$, provided only that $v \neq \equiv 1$ on $\Gamma$.

\section{The Existence of $\Delta(\Gamma, r, v ; z)$ When $g=0$}

We begin with:

Theorem 2: Suppose $\Gamma$ is an H-group of genus 0 and $\mathrm{r}$ is a real number $>2$. Then there exists a multiplier system $\mathrm{v}$ corresponding to the group $\Gamma$ and the degree $-\mathrm{r}$ with the following property:

If $\operatorname{dim} \mathrm{C}^{0}(\Gamma,-\mathrm{r}, \mathrm{v})>0$, then there is a cusp form

$\Delta(\Gamma, \mathrm{r}, \mathrm{v} ; \mathrm{z}) \epsilon \mathrm{C}^{0}(\Gamma,-\mathrm{r}, \mathrm{v})$ which has no zeros in $\mathscr{H}$. 
Proof: As before, we write

$$
\begin{aligned}
& v\left(E_{m}\right)=\exp \left(\pi i r / l_{m}+2 \pi i a_{m} / l_{m}\right), \quad 0 \leqslant a_{m} \leqslant l_{m}-1 ; 1 \leqslant m \leqslant e, \\
& v\left(P_{h}\right)=\exp \left(2 \pi i \kappa_{h}\right), \quad 0 \leqslant \kappa_{h}<1 ; 1 \leqslant h \leqslant t,
\end{aligned}
$$

with $a_{m}$ an integer. Furthermore we put

$$
\vartheta_{h}=\left\{\begin{array}{lll}
1 & \text { if } & \kappa_{h}=0 \\
0 & \text { if } & \kappa_{h}>0
\end{array}\right\}, \quad 1 \leqslant h \leqslant t
$$

Put $\mu=\operatorname{dim} C^{0}(\Gamma,-r, v)$; then by Petersson's Riemann-Roch Theorem [6, p. 194],

$$
\mu=-\sum_{h=1}^{t}\left(\vartheta_{h}+\kappa_{h}\right)+r\left(\frac{q}{2}-1+g\right)-\sum_{m=1}^{e} a_{m} / l_{m}+1
$$

where $q$ is defined by (2.9).

We want to show the existence of a multiplier system $v$ corresponding to $\Gamma$ and the degree $-r$ such that $v$ has the property (3.1). It is not too difficult to show [4, p. 273] that if $a_{m}>0$ then any element of $C^{0}(\Gamma,-r, v)$ must have a zero at the fixed point of $E_{m}$. Since such a fixed point lies in $\mathscr{H}$ any multiplier system $v$ satisfying (3.1) must be such that $a_{m}=0$ for $1 \leqslant m \leqslant e$. Accordingly we assume $a_{m}=0$ for $1 \leqslant m \leqslant e$. Petersson [6,pp. 534-535] has proved that a necessary and sufficient condition for the existence of a multiplier system $v$ corresponding to $\Gamma$ and the degree $-r$, satisfying (3.2), is that the $a_{m}$ and $\kappa_{h}$ satisfy the congruence

$$
\sum_{h=1}^{t} \kappa_{h}+\sum_{m=1}^{e} a_{m} / l_{m} \equiv r\left(\frac{q}{2}-1+g\right)(\bmod 1)
$$

Since $g=0$ here and we have assumed $a_{m}=0$ for $1 \leqslant m \leqslant e$, this congruence takes the form

$$
\sum_{h=1}^{t} \kappa_{h} \equiv r\left(\frac{q}{2}-1\right)(\bmod 1)
$$

Because $t \geqslant 1$ we can obviously choose the $\kappa_{h}, 1 \leqslant h \leqslant t$, in such a way that $\left(3.5^{\prime}\right)$ is satisfied. Alternatively, we may avoid Petersson's condition if we so desire, by simply defining $v$ on the $E_{m}$ and $P_{h}$ by means of (3.2), with all $a_{m}=0$, and the $\kappa_{h}$ chosen to be consistent with the relation

$$
\prod_{m=1}^{e} E_{m} \prod_{h=1}^{t} P_{h}=(-I)^{e+t}
$$

and the consistency condition (2.5). It is not hard to check that this procedure gives rise to a bona fide multiplier system, since $g=0$ (and thus $\Gamma$ is generated by the $E_{m}$ and the $P_{h}$ ). In fact we may arbitrarily choose $\kappa_{1}, \ldots ., \kappa_{t-1}$; then $\kappa_{t}$ is determined by the above relation. Thus if $t=1$ there is a uniquely determined multiplier system $v$ corresponding to $\Gamma$ and the degree $-r$ with $a_{m}=0$ for $1 \leqslant m \leqslant e$ and if $t>1$ there is an infinite class of such multiplier systems parametrized by the real parameters $\kappa_{1}, \ldots ., \kappa_{t-1}$.

We shall complete the proof by showing that any multiplier system $v$ with $a_{m}=0$ for $1 \leqslant m \leqslant e$ has the property (3.1). Thus assume $a_{m}=0$ for $1 \leqslant m \leqslant e, g=0$, and $\mu>0$. Then (3.4) takes the form

$$
\mu=-\sum_{h=1}^{t}\left(\vartheta_{h}+\kappa_{h}\right)+r\left(\frac{q}{2}-1\right)+1
$$


Now any function in $C^{0}(\Gamma,-r, v)$ has a zero at the parabolic cusp corresponding to $P_{h}$ of order at least $\vartheta_{h}+\kappa_{h}, 1 \leqslant h \leqslant t$. Thus if we succeed in constructing $\Delta=\Delta(\Gamma, r, v ; z) \epsilon C^{0}(\Gamma,-r, v)$ such that $\Delta$ has a zero at $i \infty$ of order $N-\sum_{h=1}^{t-1}\left(\vartheta_{h}+\kappa_{h}\right)$, with $N$ as in (2.10), then $\Delta$ will have no zeros in $\mathscr{H}$ and the proof will be complete. Let $\varphi_{1}, \ldots, \varphi_{\mu}$ be a basis for $C^{0}(\Gamma,-r, v)$ and suppose $\varphi_{j}$ has the expansion at $i \infty$

$$
\varphi_{j}(z)=\sum_{n \geqslant \vartheta_{t}} b_{n}(j) e^{2 \pi i\left(n+\kappa_{t}\right) z / \lambda}, \quad 1 \leqslant j \leqslant \mu .
$$

Form the linear combination

$$
\Delta(z)=\sum_{j=1}^{\mu} \zeta_{j} \varphi_{j}(z)=\sum_{n \geqslant \vartheta_{t}}\left(\sum_{j=1}^{\mu} \zeta_{j} b_{n}(j)\right) e^{2 \pi i\left(n+\kappa_{t}\right) z / \lambda},
$$

and note that $N-\sum_{h=1}^{t-1}\left(\vartheta_{h}+\kappa_{h}\right)=\mu-1+\vartheta_{t}+\kappa_{t}$. It suffices to solve the homogeneous equation

$$
\sum_{j=1}^{\mu} \zeta_{j} b_{n}(j)=0, \quad \vartheta_{t} \leqslant n \leqslant \mu+\vartheta_{t}-2
$$

with $\zeta_{1}, \ldots, \zeta_{\mu}$ not all zero. But this is a system of $\mu-1$ equations in $\mu$ unknowns and thus has a nontrivial solution. The proof is complete.

For each $H$-group $\Gamma$ of genus zero we want to demonstrate the existence of at least one cusp form $\Delta(\Gamma, r, v ; z)$ that is zero-free in $\mathscr{H}$. By theorem 2 it suffices to show that for each such group $\Gamma$ there exists $r>2$ and a corresponding multiplier system $v$ such that $\operatorname{dim} C^{0}(\Gamma,-r, v)>0$.

Lemma 3: Suppose $\Gamma$ is an $\mathrm{H}$-group of genus zero. If $\mathrm{e}=0$, let $\mathrm{r}=6$. If $\mathrm{e}=1, l_{1}=2$, and $\mathrm{t}=2$, choose $\mathrm{r}=8$. Otherwise put $\mathrm{r}=2 \cdot \operatorname{lcm}\left(l_{1}, \ldots, l_{\mathrm{e}}\right)$. In every case let $\mathrm{v} \equiv 1$ on $\Gamma$. Then dim $\mathrm{C}^{0}(\Gamma,-\mathrm{r}, 1)>0$.

Proof: With $r$ chosen as in the statement of the lemma we have $2 l_{m} \mid r$ for $1 \leqslant m \leqslant e$. Thus $a_{m}=0$ for $1 \leqslant m \leqslant e$. Likewise $\kappa_{h}=0$ for $1 \leqslant h \leqslant t$. Then by (3.4)

$$
\mu=\operatorname{dim} C^{0}(\Gamma,-r, 1)=-t+r\left(\frac{q}{2}-1\right)+1 .
$$

Note that in every case $r \geqslant 4$. Thus if $q \geqslant 2$ it follows that $\mu \geqslant t+2 \sum_{m=1}^{e}\left(1-\frac{1}{l_{m}}\right)-3$. In particular if $t \geqslant 4$ or $t=3$ and $e \geqslant 1$, then $\mu>0$. If $t=3$ and $e=0$, we choose $r=6$ and find that $\mu=1$.

Suppose $t=2$. Then $\mu=\frac{r}{2} \sum_{m=1}^{e}\left(1-\frac{1}{l_{m}}\right)-1$. Since $l_{m} \geqslant 2, \mu \geqslant \frac{r e}{4}-1$. With $\mathrm{e} \geqslant 2, \mu>0$ because $r \geqslant 4$. If $e=1$ and $l_{1}>2$, then again $\mu>0$. If $e=1$ and $l_{1}=2$, choose $r=8$ and $\mu=1$.

Suppose $t=1$. We apply the well-known inequality due to Siegel [12, theorem 5, p. 715]

$$
2 g-2+t+\sum_{m=1}^{e}\left(1-\frac{1}{l_{m}}\right) \geqslant 1 / 42
$$

where $\Gamma$ is an $H$-group of genus $g$. In our application here $g=0$ and $t=1$, so that (3.7) becomes

$$
\sum_{m=1}^{e}\left(1-\frac{1}{l_{m}}\right)-1 \geqslant 1 / 42
$$

From (3.8) we deduce immediately that $e \geqslant 2$. If $e \geqslant 3$, then 


$$
\mu=\frac{r}{2}\left\{\sum_{m=1}^{e}\left(1-\frac{1}{l_{m}}\right)-1\right\} \geqslant \frac{r}{2}\left(\frac{e}{2}-1\right)>0
$$

If $e=2$, then by (3.8) either $l_{1} \geqslant 3$ or $l_{2} \geqslant 3$. Thus, once more,

$$
\mu=\frac{r}{2}\left(1-\frac{1}{l_{1}}-\frac{1}{l_{2}}\right) \geqslant \frac{r}{2}\left(1-\frac{1}{2}-\frac{1}{3}\right)>0 .
$$

This completes the proof.

Combining theorem 2 and lemma 3 yields:

COROLlaRY 4: If $\Gamma$ is an H-group of genus 0 then there is an even integer $\mathrm{r} \geqslant 4$ such that $\mathrm{C}^{0}(\Gamma,-\mathrm{r}, 1)$ contains a cusp form with no zero in $\mathscr{H}$. Denote this cusp form by $\Delta(\Gamma ; \mathrm{z})$.

Examples: 1 . For $\Gamma=\Gamma(2), e=0$ and $t=3$. Thus by lemma 3 we may choose $r=6$. In this case we may choose

$$
\Delta(\Gamma ; z)=\eta^{12}(z)
$$

2. For $\Gamma=\Gamma_{\vartheta}, e=1, l_{1}=2$, and $t=2$. Thus $r=8$. In fact we have

$$
\Delta(\Gamma ; z)=\eta^{8 / 3}(2 z) \eta^{8 / 3}(z / 2) \eta^{32 / 3}\left(\frac{z+1}{2}\right) .
$$

3. For $\Gamma=\Gamma^{2}$, the subgroup of $\Gamma(1)$ generated by the squares of elements in $\Gamma(1), e=2$, with $l_{1}=l_{2}=3$, and $t=1$. Thus $r=6$ and

$$
\Delta(\Gamma ; z)=\eta^{12}(z)
$$

4. For $\Gamma=\Gamma^{3}$, the subgroup of $\Gamma(1)$ generated by the cubes of elements of $\Gamma(1), t=1$ and $e=3$, with $l_{1}=l_{2}=l_{3}=2$. Thus $r=4$ and in fact

$$
\Delta(\Gamma ; z)=\eta^{8}(z) .
$$

\section{A Special Hecke Basis Theorem}

In section 5 we present a proof of the Hecke basis theorem for the groups $\Gamma\left(\lambda_{n}\right)$ (theorem 1$)$. Here we state and prove a very special, but simple result of the same type.

THEOREM 5: Suppose $\mathrm{r}$ is a positive even integer. Then a Hecke basis for $\mathrm{C}^{0}(\Gamma,-\mathrm{r}, 1)$ exists in the following four cases:

(i) $\mathrm{g}=0, \quad \mathrm{t}=1, \mathrm{e}=3, \quad l_{1}=l_{2}=l_{3}=2$

(ii) $\mathrm{g}=0, \quad \mathrm{t}=1, \mathrm{e}=2, \quad l_{1}=2, l_{2}=3$

(iii) $\mathrm{g}=0, \quad \mathrm{t}=1, \mathrm{e}=2, \quad l_{1}=2, l_{2}=4$

(iv) $\mathrm{g}=0, \quad \mathrm{t}=1, \mathrm{e}=2, \quad l_{1}=l_{2}=3$.

REMARK: Groups with these signatures are respectively (i) $\Gamma^{3}$, (ii) $\Gamma(1)$, (iii) $\Gamma(\sqrt{2})$ and (iv) $\Gamma^{2}$.

Proof: A simple check shows that in the four cases listed the function $\Delta(\Gamma ; z)$ of corollary 4 has exactly one zero in a fundamental domain of $\Gamma$. This is a simple zero at the parabolic cusp $i \infty$. We indicate the proof in case (iii) only, the proofs in the other cases being similar. In this case $\Delta(\Gamma ; z)$ has dimension -8 . Thus we consider the functions

$$
\Delta^{l}(\Gamma ; z) \cdot E_{r-8 l}(\Gamma, 1 ; z), \quad r-8 l \geqslant 0, \quad r-8 l \neq 2, l=1,2, \ldots .
$$

Since $\operatorname{dim} C^{0}(\Gamma,-2,1)=0$, we may restrict our attention to $r \geqslant 4$. Then the number of functions given by $(4.1)$ is $[r / 8]$ if $r \not \equiv 2(\bmod 8)$ and $[r / 8]-1$ if $r \equiv 2(\bmod 8)$. Thus it suffices to prove that $\operatorname{dim} C^{0}(\Gamma,-r, 1)$ is also $[r / 8]$ or $[r / 8]-1$ according as $r \not \equiv 2(\bmod 8)$ or $r \equiv 2(\bmod 8)$. But this last fact is a simple consequence of (3.4) with the $\kappa_{h}=0$ and $g=0$. 
REMARK: It is not too hard to prove that the four cases listed in theorem 5 are the only cases in which the function $\Delta(\Gamma ; z)$ of corollary 4 has exactly one zero.

\section{Proof of Theorem 1}

For the group $\Gamma\left(\lambda_{n}\right), n \geqslant 3$, we have $g=0, t=1, e=2$, and $l_{1}=2, l_{2}=n$. We shall write $\Delta(z)$ for $\Delta\left(\Gamma\left(\lambda_{n}\right) ; z\right)$ in the remainder of the proof. If $n$ is even $\Delta(\mathrm{z})$ has degree $-2 n$ and a zero of order $(n-2) / 2$ at $i \infty$; if $n$ is odd $\Delta(z)$ has degree $-4 n$ and a zero of order $n-2$ at $i \infty$. Since $\Delta(z)$ has no zero in $\mathscr{H}$ we may form

$$
\Delta_{1}(z)= \begin{cases}\Delta(z)^{2 /(n-2)} & \text { if } n \text { is even } \\ \Delta(z)^{1 /(n-2)} & \text { if } n \text { is odd. }\end{cases}
$$

Then, in every case, $\Delta_{1}(z) \epsilon C^{0}\left(\Gamma\left(\lambda_{n}\right),-4 n /(n-2), v_{1}\right)$ where $v_{1}$ is a multiplier system corresponding to the group $\Gamma\left(\lambda_{n}\right)$ and the degree $-4 n /(n-2)$, and, in addition $\Delta_{1}(z)$ has a zero of order one at $i \infty$. Since $\Delta_{1}(i) \neq 0$, we conclude that $v_{1}(S)=1, v_{1}(T)=e^{-2 \pi i n /(n-2)}$, where

$$
S=\left(\begin{array}{ll}
1 & \lambda_{n} \\
0 & 1
\end{array}\right), \quad \lambda_{n}=2 \cos \frac{\pi}{n}, \quad \text { and } \quad T=\left(\begin{array}{rr}
0 & -1 \\
1 & 0
\end{array}\right) .
$$

We want to investigate Hecke bases for the spaces $C^{0}\left(\Gamma\left(\lambda_{n}\right),-k, 1\right)$, where $k$ is an even integer $\geqslant 4$. (Recall that $\operatorname{dim} C^{0}\left(\Gamma\left(\lambda_{n}\right),-2,1\right)=0$.)

We introduce another cusp form

$$
\Delta_{2}(z)=\Delta_{1}(z)^{k(n-2) / 4 n},
$$

which is of degree $-k$ on $\Gamma\left(\lambda_{n}\right)$ and, like $\Delta(z)$ and $\Delta_{1}(z)$, has no zeros in $\mathscr{H}$. It follows that the multiplier system $v_{2}$ connected with $\Delta_{2}(z)$ satisfies

$$
v_{2}(S)=e^{2 \pi i k(n-2) / 4 n}, \quad v_{2}(T)=e^{-\pi i k / 2} .
$$

Thus $\Delta_{2}(z)$ has multiplier system identically 1 if and only if $4 \mid k$ and $4 n \mid k(n-2)$. If these conditions hold, then $\Delta_{2}(z) \epsilon C^{0}\left(\Gamma\left(\lambda_{n}\right),-k, 1\right)$.

We consider the functions

$$
E_{k-\frac{4 n l}{n-2}}\left(\Gamma\left(\lambda_{n}\right), v_{1}^{-l} ; z\right) \cdot \Delta_{1}^{l}(z), \quad 0<l<\frac{(k-2)(n-2)}{4 n} .
$$

The inequality is imposed upon the integer $l$ to insure that the subscript $k-\frac{4 n l}{n-2}$ is greater than 2 , and thus that the Eisenstein series $E_{k-\frac{4 n l}{n-2}}$ exists in the usual sense of absolute convergence. Since $v_{1}(S)=1$ it is also true that $v_{1}^{-l}(S)=1$; consequently the function $E_{k-\frac{4 n l}{n-2}} \cdot \Delta_{1}^{l}$ of (5.2) has a zero at the cusp $i \infty$ of order precisely $l$. Thus the functions in (5.2) are linearly independent. If the conditions $4 \mid k$ and $4 n \mid k(n-2)$ hold we add $\Delta_{2}(z)$ to the set of functions (5.2). The question to be investigated is whether the resulting set of functions is a basis for $C^{0}\left(\Gamma\left(\lambda_{n}\right),-k, 1\right)$. Note that $\Delta_{2}(z)$ has a zero at $i \infty$ of order $k \cdot \frac{n-2}{4 n}>\frac{(k-2)(n-2)}{4 n}$. Thus when $\Delta_{2}(z)$ is included it is linearly independent of the functions (5.2).

Of obvious importance is the case when $4 n \mid(k-2)(n-2)$, that is, when $l=(k-2)(n-2) / 4 n$ is an integer. Then $k-4 n l /(n-2)=2$ and the Eisenstein series in question is $E_{2}\left(\Gamma\left(\lambda_{n}\right), v_{1}^{-l} ; z\right)$. By work of Petersson this Eisenstein series exists (in a sense weaker than that of absolute convergence) provided that $v_{1}^{l}$ is not identically 1 . Since $v_{1}^{l}(S)=1$, we consider

$$
v_{1}^{l}(T)=\exp \left(\frac{-2 \pi i n}{n-2} \cdot \frac{(k-2)(n-2)}{4 n}\right)=\exp \left(\frac{-\pi i(k-2)}{2}\right)
$$


Thus $v_{1}^{l}(T)=1$ if and only if $4 \mid(k-2)$. If $4 n \mid(k-2)(n-2)$, but $4 /(k-2)$, then, we have available the function $E_{2}\left(\Gamma\left(\lambda_{n}\right), v_{1}^{-l} ; z\right) \cdot \Delta_{1}^{l}(z), l=(k-2)(n-2) / 4 n$, an element of $C^{0}\left(\Gamma\left(\lambda_{n}\right),-k, 1\right)$ with zero at $i \infty$ of order exactly $(k-2)(n-2) / 4 n$.

Put $\rho(k, n)=[(k-2)(n-2) / 4 n]$. Since $4 n \mid k(n-2)$ and $4 n \mid(k-2)(n-2)$ cannot hold simultaneously there are three mutually exclusive and jointly exhaustive cases:

(i) If $4 \mid(k-2)$ and $4 n \mid(k-2)(n-2)$ we have $\rho(k, n)-1$ linearly independent functions.

(ii) If $4 \mid k$ and $4 n \mid k(n-2)$ we have $\rho(k, n)+1$ linearly independent functions.

(iii) Otherwise we have $\rho(k, n)$ linearly independent functions. that

We now turn to the calculation of $\mu(k, n)=\operatorname{dim} C^{\circ}\left(\Gamma\left(\lambda_{n}\right),-k, 1\right)$. The formula (3.4) implies

$$
\mu(k, n)=k\left(\frac{q}{2}-1\right)-a_{1} / 2-a_{2} / n, \quad q=3-\frac{1}{2}-\frac{1}{n} .
$$

Since the multiplier system here is identically $1, a_{1}=0$ or 1 according as $4 \mid k$ or $4 \mid k$, and $a_{2}$ is chosen to satisfy $a_{2} \equiv-\frac{k}{2}(\bmod n), 0 \leqslant a_{2}<n$. We have, then,

$$
\mu(k, n)=k\left(\frac{1}{4}-\frac{1}{2 n}\right)-\frac{a_{1}}{2}-\frac{a_{2}}{n},
$$

with $a_{1} \equiv-\frac{k}{2}(\bmod 2), 0 \leqslant a_{1}<2$ and $a_{2} \equiv-\frac{k}{2}(\bmod n), 0 \leqslant a_{2}<n$. The following is an immediate consequence of (5.3).

Proposition 6: $\mu(k, n)=\left[\frac{k}{4}\right]+\left[\frac{-k}{2 n}\right]$.

In order to determine when (that is, for which combinations of $k$ and $n$ ) we have constructed a complete Hecke basis for $\Gamma\left(\lambda_{n}\right)$, we now compare $\mu(k, n)$, and $\rho(k, n)$. Toward this end write $k=4 n s+2 p$, with $s$ an integer $\geqslant 0$ and $0 \leqslant p<2 n$. Then it is immediate that

and

$$
\mu(k, n)=(n-2) s+\left[\frac{p}{2}\right]-\left[\frac{-p}{n}\right]
$$

$$
\rho(k, n)=(n-2) s+\left[\frac{p-1}{2}-\frac{p-1}{n}\right],
$$

and we need only compare the numbers $\left[\frac{p}{2}\right]+\left[\frac{-p}{n}\right]$ and $\left[\frac{p-1}{2}-\frac{p-1}{n}\right]$, with $0 \leqslant p<2 n$. A straightforward calculation involving the consideration of various ranges of $p$ yields a result summarized in table 1.

The conditions $4|(k-2), 4 n|(k-2)(n-2)$ are equivalent to $4|(k-2), 2 n|(k-2)$, which are in turn equivalent to the conditions $p$ odd, $n \mid(p-1)$. Since $0 \leqslant p<2 n$, the latter two conditions hold if and only if $p=1$ or $p=n+1$ with $n$ even. Thus we have constructed $\rho(k, n)-1$ linearly independent elements of $C^{\circ}\left(\Gamma\left(\lambda_{n}\right),-k, 1\right)$ precisely when $p=1$ or $p=n+1$, with $n$ even. A glance at our table reveals that we have exactly the correct number of functions whenever $p$ is odd, and thus for odd $p$ we have constructed a Hecke basis.

Table 1

\begin{tabular}{l|c}
\hline \multicolumn{1}{c|}{ Restriction on $p$} & Value of $\mu(k, n)-\rho(k, n)$ \\
\hline$p=0$ & 1 \\
$p$ even, $0<p \leqslant n / 2+1$ & 0 \\
$p$ even, $n / 2+1<p \leqslant n$ & 1 \\
$p$ even, $n<p \leqslant \frac{3}{2} n+1$ & 0 \\
$p$ even, $\frac{3}{2} n+1<p<2 n$ & 1 \\
$p=1$ odd, $p=n+1$ & -1 \\
$p$ (i.e. $n$ even) & -1 \\
$p$ odd, $p \neq 1, p \neq n+1$ & 0 \\
\hline
\end{tabular}


The conditions $4|k, 4 n| k(n-2)$ are equivalent to $4|k, 2 n| k$, which are in turn equivalent to $p$ even, $n \mid p$. These hold if and only if $p=0$ or $p=n$ with $n$ even. Thus we have $\rho(k, n)+1$ linearly independent functions if and only if $p=0$ or $p=n$ with $n$ even. Our table shows us that when $p$ is even we have achieved a Hecke basis except when $n / 2+1<p<n$ or $\frac{3}{2} n+1<p<2 n$. In these latter two ranges of $p$ we are exactly one function short of a basis. This completes the proof of theorem 1 .

\section{The Missing Function}

It is easy to see that the "missing function" in the exceptional cases of theorem 1 is one with highest possible order zero at $i \infty$. Although in these cases we cannot express this function in the form (4.2) we can easily construct it from any given basis $\varphi_{1}, \ldots, \varphi_{\mu}$ of $C^{0}\left(\Gamma\left(\lambda_{n}\right),-k, 1\right)$. (Here we are assuming that $\mu=\operatorname{dim} C^{0}\left(\Gamma\left(\lambda_{n}\right),-k, 1\right)>0$.) In fact we give a simple and uniform construction for a cusp form in $C^{\circ}\left(\Gamma\left(\lambda_{n}\right),-k, 1\right)$ with maximal order zero at $i \infty$ that is valid in all cases, including the nonexceptional cases (those in which theorem 1 gives us a complete Hecke basis). Afterwards we show that in the nonexceptional cases the function we here construct agrees with the element of highest order zero, already constructed in the Hecke basis, up to a constant multiple.

By (3.4) we have

$$
\mu=\mu(k, n)=k\left(\frac{1}{4}-\frac{1}{2 n}\right)-\frac{a_{1}}{2}-\frac{a_{2}}{n} .
$$

On the other hand, we conclude from (2.10) that the total number of zeros of an element of $C^{0}\left(\Gamma\left(\lambda_{n}\right),-k, 1\right)$ in a fundamental domain of $\Gamma\left(\lambda_{n}\right)$ is

$$
N=k\left(\frac{1}{4}-\frac{1}{2 n}\right) \text {. }
$$

At $i$, the fixed point of $T$, a cusp form in $C^{0}\left(\Gamma\left(\lambda_{n}\right),-k, 1\right)$ has a zero of order congruent to $\frac{a_{1}}{2}$ modulo 1 ; at $\rho$, the fixed point of $S T$, such a cusp form has a zero of order congruent to $\frac{a_{2}}{n}$ modulo 1 . Thus the maximum order zero at $i^{\infty}$ of an element of $C^{0}\left(\Gamma\left(\lambda_{n}\right),-k, 1\right)$ is

$$
N-\frac{a_{1}}{2}-\frac{a_{2}}{n}=\mu(k, n)=\mu .
$$

We now proceed as in the proof of theorem 2 to form a linear combination

$$
\sum_{j=1}^{\mu} \zeta_{j} \varphi_{j}(z)=\sum_{m=1}^{\infty}\left(\sum_{j=1}^{\mu} \zeta_{j} b_{m}(j)\right) e^{2 \pi i m z / \lambda_{n}}
$$

of the basis elements $\varphi_{1}, \ldots, \varphi_{\mu}$. The homogeneous system

$$
\sum_{j=1}^{\mu} \zeta_{j} b_{m}(j), 1 \leqslant m \leqslant \mu-1
$$

has a nontrivial solution, so that the element of $C^{0}\left(\Gamma\left(\lambda_{n}\right),-k, 1\right)$ so constructed has a zero of order exactly $\mu$ at $i \infty$.

Suppose now we have $f_{1}, f_{2} \epsilon C^{0}\left(\Gamma\left(\lambda_{n}\right),-k, 1\right)$, both with zeros of maximal order $\mu$ at $i \infty$. Then both $f_{1}$ and $f_{2}$ have zeros of order $a_{1} / 2$ and $a_{2} / n$ at $i$ and $\rho$, respectively. It follows that $f_{1} / f_{2}$ is an automorphic function on $\Gamma\left(\lambda_{n}\right)$ with no poles and no zeros in a fundamental domain for $\Gamma\left(\lambda_{n}\right)$, and thus $f_{1} / f_{2}$ is a nonzero constant [3]. This shows the essential uniqueness of an element of $C^{0}\left(\Gamma\left(\lambda_{n}\right),-k, 1\right)$ with maximal order zero at $i \infty$. 


\section{Parametrization Theorems}

In this section we parametrize entire automorphic forms and multiplier systems in some special but important cases. The derivations are based on the relations $(2.10), N=r\left(g-1+\frac{q}{2}\right)$, for the total number of zeros in a fundamental region $\mathscr{R}$, of an entire form, and (3.5) $\sum_{h=1}^{t} \kappa_{h}+\sum_{m=1}^{e} a_{m} / l_{m} \equiv N \bmod 1$. (2.10) can be obtained by integrating $F^{\prime} \mid F$ around the boundary of $\mathscr{R} ;(3.5)$ is a consequence of (2.10) and definitions of the order of $F(z)$ at the points of $\mathscr{R}$. The usual way to obtain $\mu=\operatorname{dim} C^{+}(\Gamma$, $-r, v)$ is to apply Petersson's Riemann-Roch Theorem (3.4). However, this is much deeper than the above mentioned results. Some of the cases that can be handled by this method are given in the table that follows. We feel that this type of derivation may prove useful in the classroom when there is insufficient time to derive (3.4). The parametrization in the case of $\Gamma(1)$ is known [8]; however we feel that it is a very good illustration of the method. The parametrization in the case of $\Gamma_{\vartheta}$ appears to be new. Other cases which can be handled in this way are summarized in table 2 .

We consider the case of $\Gamma=\Gamma(1)$, the modular group, which has the presentation

with

$$
E_{1}=-T=\left(\begin{array}{rr}
0 & 1 \\
-1 & 0
\end{array}\right), \quad E_{2}=-T U^{-1}=\left(\begin{array}{rr}
0 & 1 \\
-1 & 1
\end{array}\right), \quad P=U=\left(\begin{array}{ll}
1 & 1 \\
0 & 1
\end{array}\right)
$$

$$
E_{1}^{2}=E_{2}^{3}=E_{1} E_{2} P=-I
$$

If $r$ is a real number and $v$ a multiplier system for $\Gamma(1)$ and $-r$, let $\kappa=\kappa_{1}, a_{1}$, and $a_{2}$ be as in section 2 . We can prove the following theorem which we might call a Hecke basis theorem for entire forms.

THEOREM 7: If $\mathrm{F} \epsilon \mathrm{C}^{+}(\Gamma(1),-\mathrm{r}, \mathrm{v})$ then there are complex constants $\mathrm{c}_{\mathrm{j}}$ such that

$$
\mathrm{F}(\mathrm{z})=\eta^{24 \kappa}(\mathrm{z}) \sum \mathrm{c}_{\mathrm{j}} \Delta^{\mathrm{j}}(\mathrm{z}) \mathrm{E}_{2 k-12 \mathrm{j}}(\mathrm{z})
$$

and the summation $\mathrm{j}$ is over the integers $0,1,2, \ldots,[2 \mathrm{k} / 12]$ with $2 \mathrm{k}-12 \mathrm{j} \neq 2$, where $2 \mathrm{k}=\mathrm{r}-12 \kappa$ is an even integer.

If we let $v_{r}$ denote the multiplier system of $\eta^{2 r}(z)$, then we can state the following:

COROLlaRY 8: With $\mathrm{F}$ expressible in the form (7.2) we have $\mathrm{v}=\mathrm{v}_{12 \kappa}$. From this it follows that

where

$$
\mathrm{v}(\mathbf{M})=\mathrm{e}[24 \kappa \Phi(\mathbf{M})], \quad \mathrm{M} \epsilon \Gamma(1),
$$

$$
\Phi(\mathbf{M})=\left\{\begin{array}{l}
\mathrm{b} / 24 \mathrm{~d}-\operatorname{sgn}(\mathrm{d}-\mathrm{l}) / 4 \quad \text { if } \quad \mathrm{c}=0 \\
\frac{1}{2}[(\mathrm{a}+\mathrm{d}) / 12 \mathrm{c}-(\operatorname{sgn} \mathrm{c}) \mathrm{s}(\mathrm{d},|\mathrm{c}|)-\operatorname{sgn} \mathrm{c} / 4] \quad \text { if } \quad \mathrm{c} \neq 0,
\end{array}\right.
$$

for $\mathrm{M}=\left(\begin{array}{ll}\mathrm{a} & \mathrm{b} \\ \mathrm{c} & \mathrm{d}\end{array}\right), \quad$ and

is Dedekind's sum [10].

$$
\mathrm{s}(\mathrm{h}, \mathrm{k})=\sum_{\mu=1}^{k-1}\left(\frac{\mu}{\mathrm{k}}-\frac{1}{2}\right)\left(\frac{\mu \mathrm{h}}{\mathrm{k}}-\left[\frac{\mu \mathrm{h}}{\mathrm{k}}\right]-\frac{1}{2}\right)
$$

This result is given in Lehner [4].

Corollary 9:

$$
\operatorname{dim} \mathrm{C}^{+}(\Gamma(1),-\mathrm{r}, \mathrm{v})=\left\{\begin{array}{lll}
{\left[\frac{\mathrm{r}}{12}-\kappa\right]+1} & \text { if } & \mathrm{r}-12 \kappa \neq 2(12) \\
{\left[\frac{\mathrm{r}}{12}-\kappa\right]} & \text { if } & \mathrm{r}-12 \kappa \equiv 2(12)
\end{array}\right.
$$

Let $\{\Gamma,-r, v\}$ denote the meromorphic automorphic forms with multiplier system $v$, and of degree $-r$. 
Corollary 10: If $\mathrm{F} \epsilon\{\Gamma(1),-\mathrm{r}, \mathrm{v}\}$, then

$$
\mathrm{F}(\mathrm{z})=\mathrm{R}(\mathrm{j}(\mathrm{z})) \mathrm{G}(\mathrm{z}),
$$

where $\mathrm{R}(\mathrm{x})$ is a rational function, $\mathrm{G} \epsilon \mathrm{C}^{+}(\Gamma(1),-\mathrm{r}, \mathrm{v})$, and $\mathrm{j}(\mathrm{z})$ is the modular invariant $\mathrm{E}_{12}(\mathrm{z}) / \Delta(\mathrm{z})$.

Proof of Theorem 7: Let $\kappa, a_{1}, a_{2}$ be as before, with $v$ a multiplier system on $\Gamma(1)$ for the degree $-r$. Then $\kappa+a_{1} / 2+a_{2} / 3 \equiv r / 12 \bmod 1$. Let $G(z)=F(z) / \eta^{24 \kappa}(z)$. Then $G \epsilon C^{+}(\Gamma(1)$, $\left.-(r-12 \kappa), v^{\prime}\right)$. Let $\kappa^{\prime}, a_{1}^{\prime}, a_{2}^{\prime}$ be the values for $v^{\prime}$ corresponding to $\kappa, a_{1}$ and $a_{2}$ for $v$. Then $\kappa^{\prime}=0$ and $a_{1}^{\prime} / 2+a_{2}^{\prime} / 3 \equiv(r-12 \kappa) / 12(\bmod 1)$. Thus $r-12 \kappa$ is an even integer $2 k$. It also follows from this relation that $v^{\prime}\left(E_{1}\right)=v^{\prime}\left(E_{2}\right)=1$. This is proved by writing $v^{\prime}\left(E_{1}\right)=e^{\pi i k+2 \pi i a_{1}^{\prime} / 2}, v^{\prime}\left(E_{2}\right)=e^{\pi i 2 k / 3+2 \pi i a_{2}^{\prime} / 3}$. Then $a_{1}^{\prime} / 2+a_{2}^{\prime} / 3 \equiv 2 k / 12 \bmod 1$ is uniquely solvable $\bmod 12$ for $a_{1}^{\prime}, a_{2}^{\prime}$ and, in fact, $2 a_{m}^{\prime}+2 k \equiv 0$ $\bmod 2(m+1), m=1,2$. For example $k=1, a_{1}^{\prime}=1, a_{2}^{\prime}=2$. Thus $v^{\prime} \equiv 1$, and therefore $G \epsilon C^{+}(\Gamma(1)$, $-2 k, 1)$. Now using Hecke's basis theorem, we find

$$
\dot{G}(z)=\sum \Delta^{j}(z) E_{2 k-12 j}(z),
$$

where summation is over $j=0,1, \ldots,[2 k / 12]$ and $2 k-12 j \neq 2$. The corollaries are immediate consequences of the theorem, the transformation formula of $\log \eta(z)$, and the well-known fact that a modular function is a rational function of $j(z)$.

As a second example we take $\Gamma$ to be $\Gamma_{\vartheta}$, the subgroup of the modular group generated by $T=\left(\begin{array}{rr}0 & -1 \\ +1 & 0\end{array}\right)$ and $U^{2}=\left(\begin{array}{ll}1 & 2 \\ 0 & 1\end{array}\right) . \Gamma_{\vartheta}$ has the presentation

$$
E=\left(\begin{array}{rr}
0 & 1 \\
-1 & 0
\end{array}\right), \quad P_{1}=\left(\begin{array}{rr}
0 & 1 \\
-1 & 2
\end{array}\right), \quad P_{2}=\left(\begin{array}{ll}
1 & 2 \\
0 & 1
\end{array}\right), \quad E^{2}=E P_{1} P_{2}=-I .
$$

Let $r$ be a real number and $v$ a multiplier system for $\Gamma_{\vartheta}$ and $-r$, and suppose

$$
v\left(P_{j}\right)=e^{2 \pi i \kappa j} \quad \text { and } \quad v(E)=e^{\pi i r / 2+2 \pi i a / 2},
$$

where $0 \leqslant \kappa_{j}<1$ and $a=0$ or 1 . Let

$$
h_{\alpha \beta}(z)=\eta^{\alpha}(2 z) \eta^{\alpha}(z / 2) \eta^{\beta}\left(\frac{z+1}{2}\right) .
$$

Then, from the transformation formula of $\log \eta(z)$,

$$
\begin{aligned}
h_{\alpha \beta}(E z) & =e((2 \alpha+\beta) / 8)(-z)^{\alpha+\beta / 2} h_{\alpha \beta}(z) \\
h_{\alpha \beta}\left(P_{1} z\right) & =e((\alpha+2 \beta) / 24)(-z+2)^{\alpha+\beta / 2} h_{\alpha \beta}(z) \\
h_{\alpha \beta}\left(P_{2} z\right) & =e((5 \alpha+\beta) / 24) h_{\alpha \beta}(z) .
\end{aligned}
$$

If we let $v_{\alpha \beta}$ denote the multiplier system for $h_{\alpha \beta}(z)$, then $h_{\alpha \beta}(z) \epsilon\left\{\Gamma_{\vartheta},-(\alpha+\beta / 2), v_{\alpha \beta}\right\}$. Let

$$
\psi(z)=h_{16 / 3,-8 / 3}(z) .
$$

By (7.11), $\psi(z) \epsilon C^{+}\left(\Gamma_{\vartheta},-4,1\right)$ and $\psi(i \infty) \neq 0, \psi(1)=0$.

LEMMA 11: (i) Let $\mathrm{r} \equiv 0 \bmod 4, \mathrm{r}>0$; then

$$
\psi^{\mathrm{j}}(\mathrm{z}) \mathrm{E}_{\mathrm{r}-4 \mathrm{j}}(\mathrm{z}, \mathrm{I}), \quad \mathrm{j}=0,1, \ldots,[\mathrm{r} / 4],
$$

is a basis for $\mathrm{C}^{+}\left(\Gamma_{\vartheta},-\mathrm{r}, 1\right)$. 
(ii) Let $r \equiv 2 \bmod 4, \mathrm{r}>2$; then

$$
\mathrm{E}_{\mathrm{r}}(\mathrm{z}, \mathrm{A}), \psi^{\mathrm{j}}(\mathrm{z}) \mathrm{E}_{\mathrm{r}-4 \mathrm{j}}(\mathrm{z}, \mathrm{I}), \mathrm{j}=0,1, \ldots,\left[\frac{\mathrm{r}}{4}\right]-1
$$

is a basis for $\mathrm{C}^{+}\left(\Gamma_{\vartheta}-\mathrm{r}, 1\right)$.

Note: The Eisenstein series $E_{2 k}(z, I)$ and $E_{2 k}(z, A)$ are defined to be the sums

$$
\frac{1}{2} \sum_{\substack{(c, d)=(0,1) \\ \bmod 2}}(c z+d)^{-2 k} \quad \text { and } \quad \frac{1}{2} \sum_{\substack{(c, d) \equiv(1,0) \\ \bmod 2}}(c z+d)^{-2 k},
$$

respectively. We use the facts that $E_{2 k}(i \infty, I)=1, E_{2 k}(1, I)=0, E_{2 k}(i \infty, A)=0, E_{2 k}(1, A)=1[9]$.

Proof: Let $F \epsilon C^{+}\left(\Gamma_{\vartheta},-r, 1\right)$. Then $F$ has $r / 4$ zeros in a fundamental region for $\Gamma_{\vartheta}$. In the case $r \equiv 0 \bmod 4$, let $c_{0}, c_{1}, \ldots ., c_{s}, s=r / 4$, be determined from the expansion of $F$ in the local variable $t=e^{2 \pi i z / 2}$ so that

$$
F(z)=\sum_{j=0}^{s} c_{j} \psi^{j}(z) E_{r-4 j}(z)
$$

has a zero of order $s+1$ at $i \infty$. But $s+1>r / 4$, so the difference is identically zero.

In the second case determine $b$ so that $G(z)=F(z)-b E_{r}(z, A)$ has a zero in the local variable $t$ at $z=1$. Then determine $c_{0}, c_{1}, \ldots ., c_{s-1}$, where $r=4 s+2$, as above, so that $H(z)=G(z)$ $-\sum_{j=0}^{s-1} c_{j} \psi^{j}(z) E_{r-4 j}(z, I)$ has a zero at $i \infty$ of order $s$ in the local variable $t=e^{2 \pi i z / 2}$. From $H(-1 / z)$ $=z^{r} H(z)$, we see that $H(i)=0$, so that $H(z)$ has order at least $1 / 2$ at $z=i$. In total $H(z)$ has at least $s+1+\frac{1}{2}>\frac{r}{4}$ zeros. Thus the difference is identically zero.

Corollary 12: $\operatorname{dim} \mathrm{C}^{+}\left(\Gamma_{\vartheta},-\mathrm{r}, 1\right)=[\mathrm{r} / 4]+1$ when $\mathrm{r}$ is an even integer larger than 2.

Theorem 13: Let $\mathrm{F}(\mathrm{z}) \epsilon \mathrm{C}^{+}\left(\Gamma_{\vartheta},-\mathrm{r}\right.$, v). Then $\mathrm{F}(\mathrm{z}) / \mathrm{h}_{\alpha \beta}(\mathrm{z}) \epsilon \mathrm{C}^{+}\left(\Gamma_{\vartheta},-(\mathrm{r}-(\alpha+\beta / 2))\right.$, 1), where $\alpha+2 \beta=24 \kappa_{1}, 5 \alpha+\beta=24 \kappa_{2}$, and $\mathrm{r}-\alpha-\frac{\beta}{2}$ is an even integer.

REMARKs: One can consider theorem 13 a Hecke basis-type theorem in view of lemma 11. For example, if $r-\alpha-\beta / 2$ is a multiple of 4 , then the elements in $C^{+}\left(\Gamma_{\vartheta},-r, v\right)$ are linear combinations of $h_{\alpha \beta}(z) \psi^{j}(z) E_{r-4 j}(z, I)$.

Proof: Let $F \epsilon C^{+}\left(\Gamma_{\vartheta},-r, v\right)$ and $\alpha$ and $\beta$ as in the statement of the theorem. Then $G=F / h_{\alpha \beta} \epsilon C^{+}\left(\Gamma_{\vartheta},-\left(r-\alpha-\beta / 2, v^{\prime}\right)\right.$. If we let $\kappa_{1}^{\prime}, \kappa_{2}^{\prime}$, and $a^{\prime}$ be as in (7.10), with $v$ replaced by $v^{\prime}$ and $r$ replaced by $r^{\prime}=r-\alpha-\beta / 2$, then $\kappa_{1}^{\prime}=\kappa_{2}^{\prime}=0$ and $a^{\prime} / 2 \equiv r^{\prime} / 4(\bmod 1)$, so $r^{\prime}$ is even. We see with with these replacements in (7.10) that $v^{\prime} \equiv 1$.

We conclude this section with a table containing parametrizations of automorphic forms and multiplier systems on various groups. The entries $E_{r}(z)$ denote Eisenstein series of degree $-r$ for the group in question. 
TABLE 2

\begin{tabular}{|c|c|c|c|c|c|}
\hline Group & Presentation & $\Delta(\Gamma, z)$ & Multiplier system $v$ for $\Gamma$ and $-r$ & Basis for $C^{+}(\Gamma,-r, v)$ & Dimension $C^{+}(\Gamma,-r, v)$ \\
\hline $\begin{array}{l}\Gamma(1) \text { modular } \\
\text { group }\end{array}$ & $\begin{array}{l}E_{1}=-T=\left(\begin{array}{rr}0 & 1 \\
-1 & 0\end{array}\right) \\
E_{2}=-T U^{-1}=\left(\begin{array}{rr}0 & 1 \\
-1 & 1\end{array}\right) \\
P=U=\left(\begin{array}{ll}1 & 1 \\
0 & 1\end{array}\right) \\
E_{1}^{2}=E_{2}^{3}=E_{1} E_{2} P=-I\end{array}$ & $\Delta(z)=\eta^{24}(z)$ & 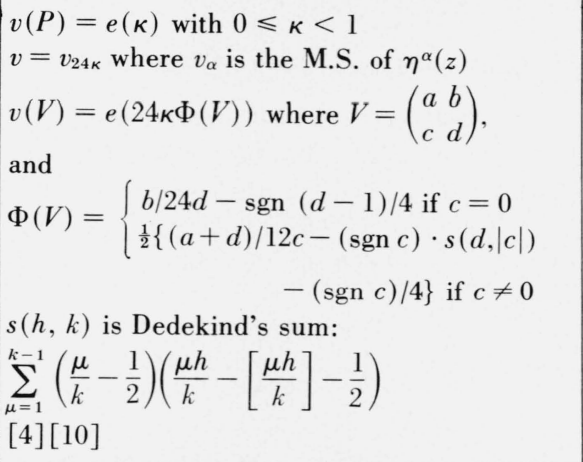 & $\begin{array}{l}\eta^{24 \kappa}(z) \Delta^{j}(z) E_{2 k-12 j}(z), \text { where } \\
\quad r=12 \kappa+2 k, \text { and } \\
\quad j=0,1, \ldots .,[2 k / 12] \\
\quad 2 k-12 j \neq 2 \\
{[8]}\end{array}$ & $\begin{array}{l}{\left[\frac{r}{12}-\kappa\right]+1 \text { if } r-12 \kappa} \\
\neq=2(12), \text { and } \\
{\left[\frac{r}{12}-\kappa\right] \text { if } r-12 \kappa \equiv 2(12) .}\end{array}$ \\
\hline $\begin{array}{l}\Gamma^{2} \\
{\left[\Gamma(1): \Gamma^{2}\right]=2}\end{array}$ & $\begin{array}{l}E_{1}=-U^{-1} T=\left(\begin{array}{rr}1 & 1 \\
-1 & 0\end{array}\right) \\
E_{2}=-T U^{-1}=\left(\begin{array}{rr}0 & 1 \\
-1 & 1\end{array}\right) \\
P=U^{2}=\left(\begin{array}{ll}1 & 2 \\
0 & 1\end{array}\right) \\
E_{1}^{3}=E_{2}^{3}=E_{1} E_{2} P=-I\end{array}$ & $\eta^{12}(z)$ & $\begin{array}{l}v(P)=e(\kappa) 0 \leqslant \kappa<1 \text { and } v_{\alpha} \text { is M.S. for } \eta^{\alpha}(z) \\
\text { then } v=v_{12 \kappa} \chi \text { where } \chi \text { is an even character } \\
\text { on } \Gamma^{2}(\chi(-I)=1)\end{array}$ & $\begin{array}{l}\text { AssumPTiON: } v \equiv 1 \text { and } r \text { even } \\
\text { integral. } \\
\eta^{12 j}(z) E_{r-6 j}(z), \text { where } \\
j=0,1, \ldots .,[r / 6], \text { and } \\
r-6 j \neq 2 .\end{array}$ & $\begin{array}{l}\text { (If } v \equiv 1, r \text { even) } \\
\qquad \begin{array}{l}{\left[\frac{r}{6}\right]+1 \text { if } r \neq \equiv 2(6), \text { and }} \\
{\left[\frac{r}{6}\right] \text { if } r \equiv 2(6)}\end{array}\end{array}$ \\
\hline $\begin{array}{l}\Gamma^{3} \\
{\left[\Gamma(1): \Gamma^{3}\right]=3} \\
\Gamma^{3} \triangleleft \Gamma(1)\end{array}$ & $\begin{array}{l}E_{1}=-U^{-1} T U=\left(\begin{array}{rr}1 & 2 \\
-1 & -1\end{array}\right) \\
E_{2}=-T=\left(\begin{array}{rr}0 & 1 \\
-1 & 0\end{array}\right) \\
E_{3}=U T U^{-1}=\left(\begin{array}{ll}-1 & 2 \\
-1 & 1\end{array}\right) \\
P=U^{3}=\left(\begin{array}{ll}1 & 3 \\
0 & 1\end{array}\right) \\
E_{1}^{2}=E_{2}^{2}=E_{3}^{2}=-I \\
E_{1} E_{2} E_{3} P=I\end{array}$ & $\eta^{8}(z)$ & $\begin{array}{l}v(P)=e(\kappa) 0 \leqslant \kappa<1 \text { and } v_{\alpha} \text { is M.S. for } \\
\eta^{\alpha}(z) \text { then } v=v_{8 \kappa} \chi \text { where } \chi \text { is an even } \\
\text { character on } \Gamma^{3} \text {. }\end{array}$ & $\begin{array}{l}\text { AssUMPTION: } v \equiv 1 \text { and } r \text { even } \\
\text { integral. } \\
\eta^{8 j}(z) E_{r-4 j}(z), \text { where } r \equiv 0(4), \\
\text { and } j=0,1, \ldots .,[r / 4] \text {, and } \\
r-4 j \neq 2 \text {. }\end{array}$ & $\begin{array}{l}\text { (If } v \equiv 1, r \text { even) } \\
\qquad \begin{array}{c}\left.\frac{r}{4}\right]+1 \text { if } r \equiv 0(4) \\
{\left[\frac{r}{4}\right] \text { if } r \equiv 2(4)}\end{array}\end{array}$ \\
\hline $\begin{array}{l}\Gamma_{\vartheta} \\
{\left[\Gamma(1): \Gamma_{\vartheta}\right]=3}\end{array}$ & $\begin{array}{l}E=-T=\left(\begin{array}{rr}0 & 1 \\
-1 & 0\end{array}\right) \\
P_{1}=-T U^{2}=\left(\begin{array}{rr}0 & 1 \\
-1 & 2\end{array}\right) \\
P_{2}=U^{2}=\left(\begin{array}{ll}1 & 2 \\
0 & 1\end{array}\right) \\
E_{1}^{2}=E P_{1} P_{2}=-I\end{array}$ & $\begin{array}{l}\zeta \eta^{8}(z) \times \\
\eta^{8}\left(\frac{z+1}{2}\right) \\
\zeta=e(-1 / 6) \\
{[9]}\end{array}$ & $\begin{array}{l}v\left(P_{j}\right)=e\left(\kappa_{j}\right) 0 \leqslant \kappa_{j}<1 j=1,2 v=v_{\alpha \beta} \\
\text { where } v_{\alpha \beta} \text { is M.S. for } \\
h_{\alpha \beta}(z)=\eta^{\alpha}(2 z) \eta^{\alpha}\left(\frac{z}{2}\right) \eta^{\beta}\left(\frac{z+1}{2}\right) \text { and } \\
\alpha+2 \beta=24 \kappa_{1}, 5 \alpha+\beta=24 \kappa_{2} . \\
v(V)=e\left(\alpha \Phi\left(V_{1}\right)+\alpha \Phi\left(V_{2}\right)+\beta \Phi\left(V_{3}\right)\right) \\
\text { where } \\
V=\left(\begin{array}{ll}a & b \\
c & d\end{array}\right), V_{3}=\left(\begin{array}{cc}a+c(b+d-a-c) / 2 \\
2 c\end{array}\right) \\
\text { and if } c \equiv 0(2)\end{array}$ & $\begin{array}{l}\text { 1) } h_{\alpha \beta}(z) E_{4}^{j}(z, A) E_{r^{\prime}-4 j}(z, I), j=0 \\
\text {. . . }\left[\frac{r^{\prime}}{4}\right]-1, \text { if } \\
r^{\prime}=r-\alpha-\beta / 2 \equiv 0(4) \\
\text { 2) } h_{\alpha \beta}(z) E_{r^{\prime}}(z, A) \\
h_{\alpha \beta}(z) E_{4}^{j}(z, A) E_{r^{\prime}-4 j}(z, I) \\
j=0, \ldots,\left[\frac{r^{\prime}}{4}\right]-1, \text { if } r^{\prime} \equiv 2(4)\end{array}$ & {$\left[\frac{r}{4}-\kappa_{1}-\kappa_{2}\right]+1$} \\
\hline
\end{tabular}


TABLE 2-Continued

\begin{tabular}{|c|c|c|c|c|c|}
\hline Group & Presentation & $\Delta(\Gamma, z)$ & Multiplier system $v$ for $\Gamma$ and $-r$ & Basis for $C^{+}(\Gamma,-r, v)$ & Dimension $C^{+}(\Gamma,-r, v)$ \\
\hline & & & $\begin{array}{l}V_{1}=\left(\begin{array}{lr}a & 2 b \\
c / 2 & d\end{array}\right), V_{2}=\left(\begin{array}{lr}a & b / 2 \\
2 c & d\end{array}\right) . \\
\text { If } c \equiv 1(2) \\
V_{1}=\left(\begin{array}{lr}2 a & b \\
c & d / 2\end{array}\right) \text { and } V_{2}=\left(\begin{array}{lr}a / 2 & b \\
c & 2 d\end{array}\right) \\
\Phi \text { defined in entry for } \Gamma(1) .\end{array}$ & & \\
\hline $\begin{array}{l}\Gamma(2) \\
{[\Gamma(1) ; \Gamma(2)]=6}\end{array}$ & $\begin{array}{l}P_{1}=-T U^{2} T=\left(\begin{array}{rr}1 & 0 \\
-2 & 1\end{array}\right) \\
P_{2}=\left(T^{-1} U^{-2}\right)^{2}=\left(\begin{array}{ll}-1 & 2 \\
-2 & 3\end{array}\right) \\
P_{3}=U^{2}=\left(\begin{array}{ll}1 & 2 \\
0 & 1\end{array}\right) \\
P_{1} P_{2} P_{3}=-I\end{array}$ & $\eta^{12}(z)$ & $\begin{array}{l}v\left(P_{j}\right)=e\left(\kappa_{j}\right) 0 \leqslant \kappa_{j}<1 j=1,2,3 \\
v=v_{\alpha \beta \gamma} \text { the M.S. for } \\
h_{\alpha \beta \gamma}(z)=\eta^{\alpha}(2 z) \eta^{\beta}\left(\frac{z}{2}\right) \eta^{\gamma}\left(\frac{z+1}{2}\right) \\
\text { where } 4 \alpha+\beta+\gamma=24 \kappa_{1}, \\
\alpha+4 \beta+\gamma=24 \kappa_{2} \text { and } \alpha+\beta+4 \gamma=24 \kappa_{3} . \\
v(V)=e\left(\alpha \Phi\left(V_{1}\right)+\beta \Phi\left(V_{2}\right)+\gamma \Phi\left(V_{3}\right)\right) \\
\text { where } V, V_{1}, V_{2}, V_{3} \text { are the same as in the } \\
\text { entry for } \Gamma_{\vartheta} \text { with } c \equiv 0(2) . \\
{[5],[13]}\end{array}$ & $\begin{array}{l}\text { Let } \psi(z)=\eta^{20 / 3}(2 z) \eta^{-4 / 3}(z / 1) \times \\
\quad \eta^{-4 / 3}\left(\frac{z+1}{2}\right) \text { and } \varphi(z) \\
\quad=\eta^{-4 / 3}(2 z) \eta^{20 / 3}(z / 2) \eta^{-4 / 3}\left(\frac{z+1}{2}\right) \cdot \\
\text { Then } \varphi, \psi \epsilon C^{+}(\Gamma(2),-2,1), \psi(i \infty) \\
\quad=0 \text { and } \varphi(0)=0 \text {. } \\
h_{\alpha \beta \gamma}(z) \psi^{m}(z) \varphi^{n}(z), \text { with } m, n \geqslant 0 \\
\text { and } 2 m+2 n=r-(\alpha+\beta+\gamma) / 2 \\
\equiv 0(2) \text {. }\end{array}$ & $r / 2-\left(\kappa_{1}+\kappa_{2}+\kappa_{3}\right)+1$ \\
\hline $\begin{array}{l}\Gamma(3) \\
{[\Gamma(1): \Gamma(3)]=24}\end{array}$ & $\begin{array}{l}-I=\left(\begin{array}{rr}-1 & 0 \\
0 & -1\end{array}\right) \\
P_{1}=U^{3}=\left(\begin{array}{ll}1 & 3 \\
0 & 1\end{array}\right) \\
P_{2}=-T U^{3} T=\left(\begin{array}{rr}1 & 0 \\
-3 & 1\end{array}\right) \\
P_{3}=A^{-1} U^{3} A=\left(\begin{array}{ll}-2 & 3 \\
-3 & 4\end{array}\right) \\
P_{4}=B^{-1} U^{3} B=\left(\begin{array}{ll}-5 & 12 \\
-3 & 7\end{array}\right) \\
(-I)^{2}=I, P_{1} P_{2} P_{3} P_{4}=I \\
A=T U^{-1}, B=T^{-1} U^{-2}\end{array}$ & $\eta^{8}(z)$ & $\begin{array}{l}v\left(P_{j}\right)=e\left(\kappa_{j}\right), 0 \leqslant \kappa_{j}<1, j=1,2,3,4 \\
v=v_{\alpha \beta \gamma \delta} \text { the M.S. for } \\
h_{\alpha \beta \gamma \delta}(z)=\eta^{\alpha}(3 z) \eta^{\beta}\left(\frac{z}{3}\right) \eta^{\gamma}\left(\frac{z+1}{2}\right) \eta^{\delta}\left(\frac{z+2}{3}\right) \\
\text { where }-4 \alpha=-11 \kappa_{1}+\kappa_{2}+\kappa_{3}+\kappa_{4}, \\
-4 \beta=\kappa_{1}-11 \kappa_{2}+\kappa_{3}+\kappa_{4}, \\
-4 \gamma=\kappa_{1}+\kappa_{2}-11 \kappa_{3}+\kappa_{4} \text { and } \\
-4 \delta=\kappa_{1}+\kappa_{2}+\kappa_{3}-11 \kappa_{4} . \\
{[13]}\end{array}$ & $\begin{array}{l}\text { Let } \psi(z)=h_{11 / 4,-1 / 4,-1 / 4,-1 / 4}(z) \text { and } \\
\varphi(z)=h_{-1 / 4,11 / 4,-1 / 4,-1 / 4}(z) \in C^{+}(\Gamma(3), \\
-1, \chi) \text { where } \chi= \pm 1, \chi\left(P_{i}\right)=1 \\
\chi(-I)=-1 . \text { Then } \psi(i \infty)=0 \\
\varphi(0)=0 . \\
h_{\alpha \beta \gamma \delta}(z) \varphi^{m}(z) \psi^{n}(z) \text { where } m, n \geqslant 0 \\
\text { and } m+n=r-\kappa_{1}-\kappa_{2}-\kappa_{3}-\kappa_{4} .\end{array}$ & $r-\kappa_{1}-\kappa_{2}-\kappa_{3}-\kappa_{4}+1$ \\
\hline$\Gamma(\sqrt{2})$ & $\begin{array}{l}E_{1}=\left(\begin{array}{rr}0 & 1 \\
-1 & 0\end{array}\right) \\
E_{2}=\left(\begin{array}{rr}0 & 1 \\
-1 & \sqrt{2}\end{array}\right) \\
P=\left(\begin{array}{rr}1 & \sqrt{2} \\
0 & 1\end{array}\right) \\
E_{1}^{2}=E_{2}^{4}=-I=E_{1} E_{2} P \\
V=\left(\begin{array}{lr}a & b \sqrt{2} \\
c \sqrt{2} & d\end{array}\right) \begin{array}{l}\text { called } \\
\text { even }\end{array} \\
V=\left(\begin{array}{lr}a \sqrt{c} & b \\
c & d \sqrt{2}\end{array}\right) \begin{array}{l}\text { called } \\
\text { odd }\end{array}\end{array}$ & $\begin{array}{l}\eta^{8}(\sqrt{2} z) \times \\
\eta^{8}(z / \sqrt{2})\end{array}$ & $\begin{array}{l}v(V)=v_{s, t} v(V, \sqrt{2},-r) \text { where } \\
v(V, \sqrt{2},-r) \text { is M.S. for } \\
\quad[\eta(\sqrt{2} z) \eta(z / \sqrt{2})]^{r} \\
\quad \text { If } V \text { is even } \\
v_{s, t}(V)=e((b d-a c+2 b c) t / 4), \text { and } \\
v(V, \sqrt{2},-r)=e\left(-r \operatorname{sgn} \frac{c}{4}+r \Phi\left(V_{1}\right)+r \Phi\left(V_{2}\right)\right) \\
\text { where } V_{1}=\left(\begin{array}{lr}a & 2 b \\
c & d\end{array}\right) \text { and } V_{2}=\left(\begin{array}{ll}a & b \\
2 c & d\end{array}\right) \\
\Phi \text { is defined in the entry for } \Gamma(1) \\
\text { If } V \text { is odd } \\
v_{s, t}(V)=e(s+t(a c-b d-2 a d) / 4), \text { and }\end{array}$ & $\begin{array}{l}\nu(P)=e(\kappa), r^{\prime}=r-8 \kappa \\
\eta^{8 \kappa}(z, \sqrt{2}) \eta^{8 j}(z, \sqrt{2}) E_{r^{\prime}-8 j}\left(z, v_{0,0}\right) \\
\text { when }\left(a_{1}, a_{2}\right)=(0,0),(1,3) \\
\quad(0,2) \text { or }(1,1) \\
\eta^{8 \kappa}(z, \sqrt{2}) \eta^{8 \kappa}(z, \sqrt{2}) E_{r^{\prime}-8 j}\left(z, v_{1,0}\right) \\
\text { when }\left(a_{1}, a_{2}\right)=(1,2),(0,1) \\
\quad(1,0),(0,3) \\
\text { where } v\left(E_{1}\right)=e^{\pi i r / 2+2 \pi i a_{1} / 2} \\
\quad v\left(E_{2}\right)=e^{\pi i r / 4+2 \pi i a_{2} / 4}, \\
\text { and } j=0,1, \ldots,\left[\frac{r^{\prime}}{8}\right], r^{\prime}-8 j \neq 2 . \\
{[14]}\end{array}$ & $\begin{array}{l}{\left[\frac{r}{8}-\kappa\right]+1 \text { when }} \\
r^{\prime}=r-8 \kappa \neq 2(4) \\
{\left[\frac{r}{8}-\kappa\right] \text { if } r^{\prime} \equiv 2(4)} \\
\text { and }\left(a_{1}, a_{2}\right)=(1,3) \\
\text { The case }\left(a_{1}, a_{2}\right)=(0,1) \text { is } \\
\text { excluded in the above } \\
\text { formula. }\end{array}$ \\
\hline
\end{tabular}




\begin{tabular}{|c|c|c|c|c|c|}
\hline & & & $\mid \begin{array}{l}v(V,-\sqrt{2},-r)=e\left(-r \operatorname{sgn} \frac{c}{4}+r \Phi\left(V_{3}\right)\right. \\
\left.\quad+r \Phi\left(V_{4}\right)\right) \\
\text { where } V_{3}=\left(\begin{array}{ll}2 a & b \\
c & d\end{array}\right) \text { and } V_{4}=\left(\begin{array}{ll}a & b \\
c & 2 d\end{array}\right) . \\
{[2],[14]}\end{array}$ & & \\
\hline$\Gamma(\sqrt{3})$ & $\begin{array}{l}E_{1}=\left(\begin{array}{rr}0 & 1 \\
-1 & 0\end{array}\right) \\
E_{2}=\left(\begin{array}{rr}0 & 1 \\
-1 & \sqrt{3}\end{array}\right) \\
P=\left(\begin{array}{ll}1 & \sqrt{3} \\
0 & 1\end{array}\right) \\
E_{1}^{2}=E_{2}^{6}=-I=E_{1} E_{2} P \\
V=\left(\begin{array}{rr}a & b \sqrt{3} \\
c \sqrt{3} & d\end{array}\right) \text { even } \\
V=\left(\begin{array}{rr}a \sqrt{3} & b \\
c & d \sqrt{3}\end{array}\right) \text { odd }\end{array}$ & $\begin{array}{l}\eta^{12}(\sqrt{3} z) \times \\
\eta^{12}(z / \sqrt{3})\end{array}$ & $\begin{array}{l}v(V)=v_{s, t}(V) v(V, \sqrt{3},-r) \text { where } \\
v(V, \sqrt{3},-r) \text { is M.S. for } \\
\quad[\eta(\sqrt{3} z) \eta(z / \sqrt{3})]]^{r} . \\
\quad \text { If } V \text { is even } \\
v_{s, t}(V)=e(t(b d-a c+3 b c) / 6), \text { and } \\
v(V)=e\left(-r \operatorname{sgn} \frac{c}{4}+r \Phi\left(V_{1}\right)+r \Phi\left(V_{2}\right)\right) . \\
\quad \text { If } V \text { is odd } \\
v_{s, t}(V)=e\left(\frac{s}{2}+t(a c-b d+3 a d) / 6\right), \\
\text { and } \\
v(V, \sqrt{3},-r)=e\left(-r \operatorname{sgn} \frac{c}{4}+r \Phi\left(V_{3}\right)\right. \\
\left.\quad+r \Phi\left(V_{4}\right)\right) \\
\text { where } V_{1}, V_{2}, V_{3}, V_{4} \text { are as in entry for } \\
\quad \Gamma(\sqrt{2}) \text { with } 3 \text { replacing } 2 . \\
{[2],[14]}\end{array}$ & $\begin{array}{l}v(P)=e(\kappa), r^{\prime}=r-6 \kappa \\
\text { 1) } r^{\prime} \equiv 0(2), \eta^{6 \kappa}(z, \sqrt{3}) \eta^{6 j}(z, \\
\sqrt{3}) E_{r^{\prime}-6 j}\left(z, v_{s-j, 0}\right) \\
\text { where } s \equiv r^{\prime} / 2+a_{1} \bmod 2 \text { and } \\
v\left(E_{1}\right)=e^{2 \pi i r^{\prime} / 4+2 \pi i a 1 / 2}, \\
\text { 2) } r^{\prime} \equiv 1(2), \eta^{6 \kappa}(z, \sqrt{3}) \eta^{6 j}(z, \\
\sqrt{3}) E_{r^{\prime}-6 j}\left(z, v_{1} v_{s-j, 5}\right) \\
\text { where } s \equiv\left(r^{\prime}-1\right) / 2+a_{1}(2), \\
a_{1} \text { as above and } v_{1} \text { is the M.S. } \\
\text { for } \eta(z, \sqrt{3}) \\
{[14]}\end{array}$ & $\begin{array}{l}{\left[\frac{r}{6}-\kappa\right]+1 \text { if } r^{\prime}=r-6 \kappa} \\
\neq 2(6), \\
\text { and }\left[\frac{r}{6}-\kappa\right] \text { if } r^{\prime} \equiv 2(6) .\end{array}$ \\
\hline
\end{tabular}




\section{References}

[1] Hecke, E., Über Modulfunktionen und die Dirichletschen Reihen mit Eulerscher Produktentwicklung I, Math. Ann. 114, 1-56 (1937).

[2] Knopp, M. I., Determination of certain roots of unity in the theory of automorphic forms of dimension zero, Duke Math, J. 27, 497-506 (1960).

[3] Knopp, M. I., Lehner, J., and Newman, M., A bounded automorphic form of dimension zero is a constant, Duke Math. J. 32, 457-460 (1965).

[4] Lehner, J., Discontinuous Groups and Automorphic Functions, Amer. Math. Soc. Math. Surveys VIII, Providence (1964).

[5] Maak, W., Fastperiodische Funktionen auf der Modulgruppe, Math. Scand. 3, 44-48 (1955).

[6] Petersson, H., Zur analytischen Theorie der Grenzkreisgruppen II, III, Math. Ann. 115, 175-204, 518-572 (1938).

[7] Petersson, H., Automorphe Formen als metrische Invarianten I, Math. Nachr. 1, 158-213 (1948).

[8] Petersson, H., Die linearen Relationen zwischen den ganzen Poincaréschen Reihen von reeler Dimension zur Modulgruppe, Abh. Math. Sem. Hamburg Univ. 12, 415-472 (1938).

[9] Petersson, H., Uber die Eisensteinschen Riehen der Thetagruppe, Abh. Math. Sem. Univ. Hamburg 31, $166-178$ (1967).

[10] Rademacher, H., On the transformation of $\log \eta(\tau)$, J. Indian Math. Soc. 19, 25-30 (1955).

[11] Rademacher, H., and Zuckerman, H. S., On the Fourier coefficients of certain modular forms of positive dimension, Annals of Math. 39, 433-462 (1938).

[12] Siegel, C. L., Some remarks on discontinuous groups, Annals of Math. 46, 708-718 (1945).

[13] Smart, J. R., On modular forms of levels two and three, Michigan Math. J. 9, 233-239 (1962).

[14] Smart, J. R., Parametrization of automorphic forms for the Hecke groups $G(\sqrt{2})$ and $G(\sqrt{3})$, Duke Math. J. 31, 395-404 (1964).

(Paper 74B3-325) 